INPLASY

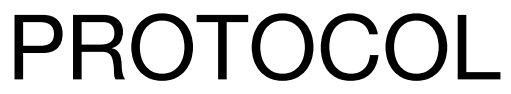

To cite: Hwang et al.

Acupuncture treatment for emotional problems in infertile women: a protocol for systematic review and metaanalysis. Inplasy protocol

202150082. doi:

10.37766/inplasy2021.5.0082

Received: 21 May 2021

Published: 21 May 2021

Corresponding author:

Jang-Kyung Park

vivat314@pusan.ac.kr

Author Affiliation:

Pusan National University

Support: No.

NRF-2019R1G1A1100022.

Review Stage at time of this submission: The review has not yet started.

Conflicts of interest:

None declared.

\section{Acupuncture treatment for emotional problems in infertile women: a protocol for systematic review and meta-analysis}

\author{
Hwang, SI1; Sung, SH²; Yoon, YJ3; Park, JK4.
}

Review question / Objective: To evaluate the efficacy and safety of acupuncture for treating emotional problems in infertile women.

Condition being studied: Infertile women with emotional problems such as anxiety, depression, low self-efficacy, distress, fear, panic, and nervousness.

Information sources: The following electronic databases will be searched from their inception to April 30, 2021: MEDLINE, EMBASE, Cochrane Library, Korean Medical Databases (KoreaMed, Korean studies Information Service System, Korean Traditional Knowledge Portal, Oriental Medicine Advanced Searching Integrated System, Research Information Sharing Service, and National Digital Science Library), and Chinese databases (CNKI and Wan Fang Database).

INPLASY registration number: This protocol was registered with the International Platform of Registered Systematic Review and Meta-Analysis Protocols (INPLASY) on 21 May 2021 and was last updated on 21 May 2021 (registration number INPLASY202150082).

\section{INTRODUCTION}

Review question / Objective: To evaluate the efficacy and safety of acupuncture for treating emotional problems in infertile women.

Condition being studied: Infertile women with emotional problems such as anxiety, depression, low self-efficacy, distress, fear, panic, and nervousness.

\section{METHODS}

Participant or population: This study will include women diagnosed with infertility and emotional problems. These emotional 
problems include anxiety, depression, low self-efficacy, distress, fear, panic, and nervousness. There will be no restrictions on age, race, nationality, education, or economic status.

Intervention: Any acupuncture type will be accepted, including acupuncture, acupressure, electroacupuncture, auricular acupuncture, scalp acupuncture, hand acupuncture, pharmacopuncture, and transcutaneous electric acupoints. Control interventions will include placebo/sham acupuncture, no treatment, or conventional treatment. RCTs on the combined effects of acupuncture and conventional treatment will be included if identical conventional treatment was applied to both groups.

Comparator: The following treatment comparisons will be considered. 1) Acupuncture versus placebo/sham acupuncture 2) Acupuncture versus no treatment 3) Acupuncture versus conventional treatment 4) Acupuncture plus conventional treatment versus identical conventional treatment alone.

Study designs to be included: Randomized controlled trials.

Eligibility criteria: All randomized controlled trials (RCTs) evaluating the acupuncture treatment effect on emotional problems in infertile women will be included. Other studies, including non-RCTs, case series, case reports, crossover studies, letters, or laboratory studies, will be excluded. Study eligibility will not be restricted by language or publication date.

Information sources: The following electronic databases will be searched from their inception to April 30, 2021: MEDLINE, EMBASE, Cochrane Library, Korean Medical Databases (KoreaMed, Korean studies Information Service System, Korean Traditional Knowledge Portal, Oriental Medicine Advanced Searching Integrated System, Research Information Sharing Service, and National Digital Science Library), and Chinese databases (CNKI and Wan Fang Database).
Main outcome(s): 1) Emotion-related assessment scales (e.g. Hamilton Anxietyrating Scale, State-trait Anxiety Inventory, Amsterdam Preoperative Anxiety and Information Scale, Self-rating Depression Scale, The Fertility Problem Inventory, Perceived Stress Scale, and Infertility SelfEfficacy scale).

Additional outcome(s): 1) Total effectiveness rate for emotional problems; 2) Quality of life; 3) Clinical pregnancy rate; 4) Adverse events.

Quality assessment / Risk of bias analysis: Two review authors will independently evaluate the risk of bias using the Cochrane risk of bias assessment tool. The following domains will be assessed: random sequence generation, allocation concealment, blinding of participants, blinding of outcome assessors, incomplete outcome data, selective outcome reporting, and other sources of bias (including factors likely to influence the results, such as extreme baseline imbalance of age, comorbidity, onset, or physical conditions). The risk of bias will be classified into three levels: low, high, and unclear.

Strategy of data synthesis: For metaanalysis, RevMan Version 5.4 software will be used to combine the relative risks for dichotomous outcomes and mean differences or standardized mean differences for continuous outcomes, with both having $95 \%$ confidence intervals. We will pool data across the studies for metaanalysis using random-effect or fixed-effect models.

Subgroup analysis: If data are available, subgroup analysis will be conducted based on the types of acupuncture treatment (e.g., acupuncture, electroacupuncture, pharmacopuncture, or scalp acupuncture) and emotional problems.

Sensitivity analysis: Sensitivity analysis will be performed to determine the robustness of the review results with respect to the following aspects: impact of sample size, 
effect of missing data, and methodological quality.

Country(ies) involved: Republic of Korea.

Keywords: infertility-related emotional problems, infertility, infertile women, acupuncture, protocol, systematic review, meta-analysis.

Contributions of each author:

Author 1 - Su-In Hwang.

Email: hwangsi1216@gmail.com

Author 2 - Soo-Hyun Sung.

Email: koyote10010@nikom.or.kr

Author 3 - Young-Jin Yoon.

Email: yyj@pusan.ac.kr

Author 4 - Jang-Kyung Park.

Email: vivat314@pusan.ac.kr 